\title{
BMJ Open 'Screen and Treat for Anaemia Reduction (STAR)' strategy: study protocol of a cluster randomised trial in rural Telangana, India
}

\author{
Bharati Kulkarni (D) , ${ }^{1}$ Little Flower Augustine, ${ }^{1}$ Raghu Pullakhandam, ${ }^{2}$ \\ Anju Sinha Pradhan (D) , ${ }^{3}$ Teena Dasi, ${ }^{1}$ Ravindranadh Palika, ${ }^{2}$ \\ Santosh Kumar Banjara, ${ }^{1}$ Harshpal Singh Sachdev ${ }^{4}$
}

To cite: Kulkarni B, Augustine LF, Pullakhandam R, et al. 'Screen and Treat for Anaemia Reduction (STAR)' strategy: study protocol of a cluster randomised trial in rural Telangana, India. BMJ Open 2021;11:e052238. doi:10.1136/ bmjopen-2021-052238

- Prepublication history and additional supplemental material for this paper are available online. To view these files, please visit the journal online (http://dx.doi.org/10.1136/ bmjopen-2021-052238).

Received 10 April 2021 Accepted 03 December 2021

Check for updates

(c) Author(s) (or their employer(s)) 2021. Re-use permitted under CC BY-NC. No commercial re-use. See rights and permissions. Published by BMJ.

${ }^{1}$ Clinical Division, ICMR-National Institute of Nutrition, Hyderabad Telangana, India

${ }^{2}$ Drug Safety Division, ICMRNational Institute of Nutrition, Hyderabad, Telangana, India

${ }^{3}$ Reproductive and Child Health, Indian Council of Medical Research, New Delhi, Delhi, India

${ }^{4}$ Department of Paediatrics, Sitaram Bhartia Institute of Science and Research, New Delhi, Delhi, India

Correspondence to

Dr Bharati Kulkarni;

dr.bharatikulkarni@gmail.com

\section{ABSTRACT}

Introduction Current anaemia control programme focusing on prophylactic iron supplementation and facility-based screening with haemoglobin estimation is inadequate to reduce the high prevalence of anaemia in India. This study aims to examine the impact of community level 'screen and treat' strategy for increasing population mean haemoglobin and reducing anaemia prevalence in the rural population.

Methods and analysis An open-labelled cluster randomised controlled trial will be conducted in rural areas of Medchal district, Telangana, India. All individuals served by one Accredited Social Health Activist (ASHA) constitute one cluster and will be randomised in the ratio of $1: 1$ by covariate constrained randomisation. Eligible members aged between 6 months and 50 years (men, women, children and adolescents) will be included in the study. Intervention group will be screened for anaemia using a point of care haemoglobin estimation followed by treatment with iron-folic acid for 3 months. The intervention delivered by the ASHAs will be supported by an electronic decision support system and simplified medication regimen. Educational videos and interactive voice response system will be used to enhance compliance. The control group will continue to receive benefits of ongoing anaemia control programmes but there will be no active intervention by the study team. At 6 months, haemoglobin will be measured in participants from both arms. The primary outcome will be the difference in population mean haemoglobin in two arms and the secondary outcome will be the difference in the anaemia prevalence in two arms among 6-59 months old children. Multilevel models will be used for analysis accounting for data clustering.

Ethics and dissemination The study is approved by the institutional ethics committee of National Institute of Nutrition, Hyderabad. The results will be published in peer-reviewed journals and disseminated to policymakers. Findings will also be shared with study participants and community leaders.

Trial registration number CTRI/2019/01/016918.

\section{INTRODUCTION}

Anaemia is one of the major nutritional public health problems in developing countries with
Strengths and limitations of this study

- Point of care screening for anaemia with a precise automated method of haemoglobin estimation followed by appropriate treatment is likely to have a wider reach and substantial reduction in anaemia.

- Novel components such as customised software for coordinating study activities and simplifying treatment delivery by Accredited Social Health Activists (ASHAs), innovative methods of behaviour change communication are likely to enhance efficiency of the intervention delivery.

- A theory-based process evaluation and assessment of cost-effectiveness embedded in the study will provide important information for effective scale up of the intervention.

- Non-availability of baseline population mean haemoglobin in the control group due to the nature of the intervention will be a limitation; so comparison of the baseline anaemia prevalence in the two arms will not be possible.

- Logistic challenges may arise in the intervention delivery by the ASHAs who have varying responsibilities depending on the ongoing and new government programmes.

adverse consequences including increased morbidity and mortality in women and children, impaired cognition in children and decreased physical work capacity in adults. ${ }^{1}$ Anaemia prevalence in India continues to be high despite ongoing anaemia control programmes for almost five decades. National surveys like Comprehensive National Nutrition Survey (2016-2018) and National Family Health Survey (NFHS)-4 (2015-2016) report prevalence of anaemia to be about $40 \%$ in under five children and $53 \%$ in women of reproductive age. ${ }^{23}$

In response to the challenge of persistent widespread anaemia in India, the efforts to strengthen the anaemia control programmes 
to date included increase in the iron-folic acid (IFA) dosages, inclusion of additional beneficiary groups using a life course approach and measures to increase compliance such as making the tablets enteric coated to reduce side effects. ${ }^{3}{ }^{4}$ The limited success of these measures prompts re-examination of the current strategies of anaemia control.

Under the currently ongoing anaemia control programme, a large proportion of anaemic individuals receive only prophylactic doses of IFA that are inadequate for treatment and recovery from anaemia. Only a very small proportion of anaemic persons reach the health facilities for haemoglobin estimation and are likely to receive appropriate treatment following diagnosis of anaemia. The universal prophylactic IFA supplementation approach, therefore, may not be able to deliver the desired benefits. Population level screening using point of care (POC) or on-site testing method followed by appropriate treatment for anaemia, hereafter referred to as 'screen and treat for anaemia reduction (STAR) strategy' is likely to lead to greater reduction in anaemia prevalence compared with the current practice of universal IFA supplementation. Guidelines of recently launched Anaemia Mukt Bharat (anaemia-free India) campaign advocate 'test and treat' approach with focus on pregnant women and adolescent girls and boys (10-19 years). ${ }^{4}$ The programme recommends use of POC devices like digital haemoglobinometers (invasive for pregnant women and non-invasive for adolescents) for diagnosis of anaemia followed by treatment as per the grade of anaemia. However, these POC devices are known to have high systematic and random errors. ${ }^{5}$ Evidence on the feasibility, impact and cost-effectiveness of implementing STAR strategy using a robust validated POC method for haemoglobin estimation is currently lacking.

A few quasi-experimental pre-post studies have reported substantial reduction in anaemia prevalence following screening and treatment of anaemia. ${ }^{67}$ The evidence based on randomised controlled trials (RCTs) is, however, not available. A study conducted at our centre in women of reproductive age showed almost $40 \%$ reduction in anaemia (from $70 \%$ at baseline to $30 \%$ ) after screening followed by IFA treatment for 3 months. ${ }^{8}$ The available evidence is not adequate to guide large scale anaemia control programmes. The present trial aims to bridge this important knowledge gap by implementing the STAR strategy at community level and evaluating its impact on mean population haemoglobin compared with the currently operational anaemia control programme in a cluster RCT. Moreover, the study includes additional components of process evaluation and cost-effectiveness analysis to guide the scaling up of the intervention.

\section{Hypothesis and objectives}

We hypothesise that population level implementation of STAR strategy will lead to higher mean population haemoglobin by $0.25 \mathrm{SD}$ as compared with the control arm with existing anaemia control programme.

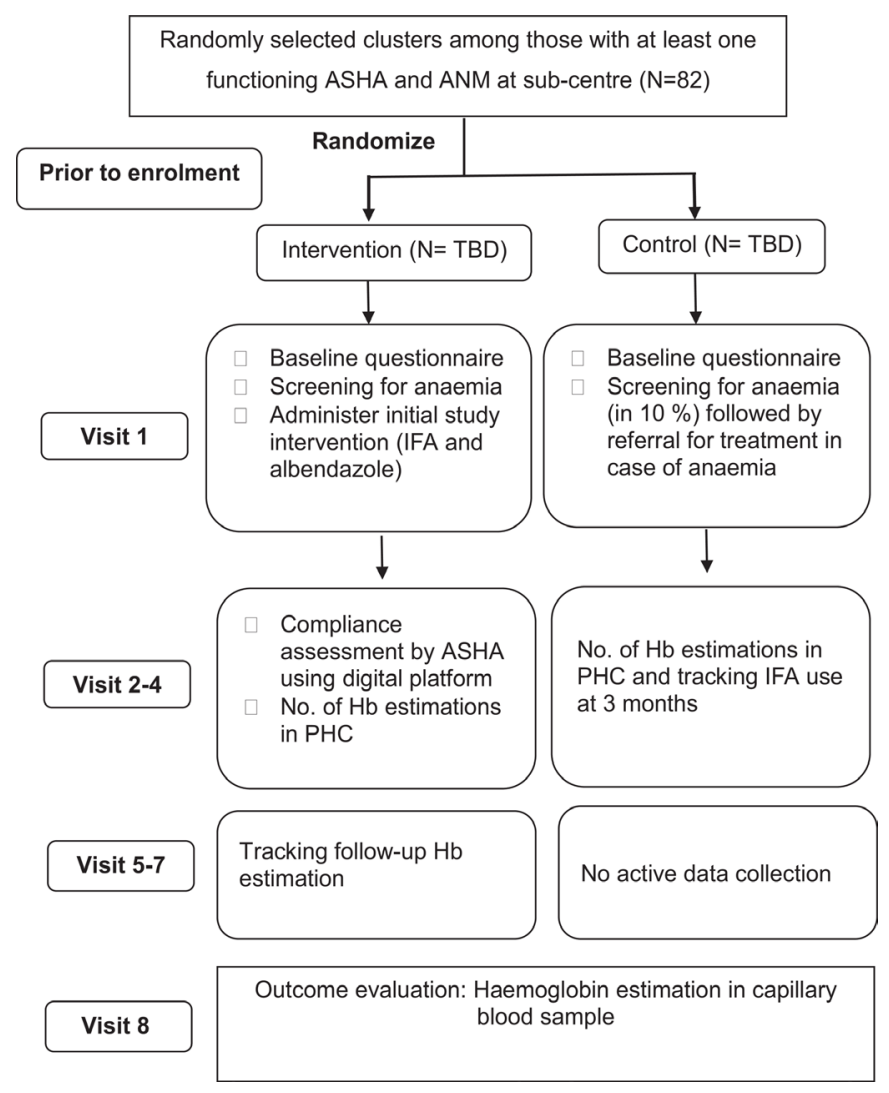

Figure 1 Flow diagram of activities: cluster randomised trial. ANM, auxiliary nurse and midwife; ASHA, Accredited Social Health Activist; IFA, iron-folic acid; Hb, haemoglobin; PHC, primary health centre.

The primary objective is to determine the impact of population level STAR intervention on mean population haemoglobin compared with the existing anaemia control programme. The intervention is likely to result in substantial anaemia reduction in population groups like under five children who have high anaemia prevalence. Our main secondary objective is comparison of the anaemia prevalence among 6-59 months old children in the two study arms. This group is chosen because of the public health importance of anaemia reduction in this age group. Additional secondary objectives are: (1) to compare the process measures related to the implementation and delivery of the STAR strategy with those measures in the control arm; and (2) to evaluate the costeffectiveness of the STAR strategy as compared with the existing anaemia control programme. Detailed protocols for the process evaluation and cost-effectiveness evaluation will be reported separately.

\section{METHODS}

\section{Study design}

The STAR trial (protocol V.2, dated 12 May 2020) is planned as a two arm (1:1) open-labelled cluster randomised superiority trial with a parallel process and cost-effectiveness evaluation (figure 1). Masking the participants or the project staff is not possible due to the 
nature of the intervention. The unit of randomisation is a cluster of household members covered by a frontline health worker called Accredited Social Health Activist (ASHA).The ASHAs are residents of the village they serve, are literate and receive performance-based incentives for their activities such as mobilising community for immunisation, antenatal care and IFA supplementation to the beneficiaries.

\section{Patient and public involvement}

The study participants were not involved in development of the study design or objectives but the focus group discussions in the formative phase have helped optimisation of study procedures. Reports of the blood investigations conducted with autoanalyser will be shared with the participants.

\section{Study setting}

The study will be carried out in Medchal district of Telangana State, India, with predominantly rural population $(61 \%)$. The prevalence of anaemia among the rural children (6-59 months) and non-pregnant women in this district was $73.8 \%$ and $57.4 \%$, respectively, as per the NFHS-5 (district level data) in year 2019-20209. These prevalence estimates are similar to the average anaemia prevalence in Telangana state for the respective groups. The proportion of pregnant women who reported consuming IFA for at least 100 days $(61 \%)$ is also similar to the state average (58\%). Comparative district level estimates on anaemia prevalence or IFA intake are not available for NFHS-5. From Medchal district, a total of 57 villages which are served by three rural primary health centres (PHCs) were contacted. Out of these, 47 villages, each with a functional ASHA in the village and auxiliary nurse and midwife at the corresponding subcentre formed the sampling frame of our study. The participant enrolment has started in July 2021. The planned end date for the study is December 2022.

\section{Study participants, inclusion and exclusion criteria}

All the household members listed in the registers maintained by the ASHA workers will be screened. Children (above 6 months), adolescents and adults below 50 years of age will be eligible to participate in the study. Although as per the National Rural Health Mission norms, one ASHA covers 1000 population, this number varies due to various contextual reasons. The population sizes of the 47 clusters in the sampling frame are provided in online supplementary table 1. Individuals with febrile illnesses (except those with malaria) will be included if the fever subsides by the time of second visit after 7 days.

Individuals suspected to have illnesses where iron supplementation is contraindicated (ie, those reporting history of repeated blood transfusion, known cases of haemolytic anaemia, chronic diarrhoea, stomach ulcer, heart surgery/prosthetic valves, cancer, tuberculosis; those who are on medications like tetracycline or anticoagulants; and women in first trimester of pregnancy) will be excluded from the study. Those who are planning to migrate within 6 months will also be excluded from the study.

\section{Sample size}

In the absence of effect size estimates based on previous reports of similar interventions, we considered a conservative expected difference of $0.25 \mathrm{SD}$ in the population mean haemoglobin in the two arms at the end of the study. With $95 \%$ CI, $90 \%$ power and with SD of $26 \mathrm{~g} / \mathrm{L}$ (based on District Level Health Survey-4 data for the state of Telangana),${ }^{10}$ the required sample size is estimated to be 337 per arm in case of individual randomisation. To detect $12 \%$ change in anaemia status among children, a sample size of 267 in each arm is estimated. To calculate the design effect to account for clustering, the available intracluster correlation (ICC) values (table 1) based on previous studies with small clusters $(\mathrm{n} \approx 25-38)$ may not be applicable to the large clusters in our study. We will therefore derive the ICC and calculate the final sample size based on an internal pilot study in six clusters.

\section{Study procedures}

Participant enrolment, randomisation, intervention allocation

The households will be enumerated and geocoded through door-to-door visit by study staff who will be accompanied by the ASHA. All the ASHAs from the selected clusters will be randomised to either of the two arms by computer generated random numbers using covariate constrained randomisation by an independent statistician.

\section{Covariate constrained randomisation}

Covariate constrained randomisation will be used to attain balance in participant characteristics between the two arms. The covariates which can influence the population mean haemoglobin and facility-based screening or intervention are considered for randomisation. The covariates include households per cluster (maximum permissible difference of 500 households), population proportion of $0-6$ years children (difference of $<5 \%$ ) and distance to nearest health facility (difference of $<10$ $\mathrm{km})$. The least distance between the centre point of the village and the nearest health facility ( $\mathrm{PHC} /$ community health centre) measured by high sensitivity Wide Area Augmentation System) enabled GPS (Global Positioning System) receiver (Garmin eTrex $20 \times / 30 \times$ ) will be used.

\section{Interventions and intervention delivery}

\section{POC screening and treatment of anaemia}

Participants will be screened for anaemia near their household (within a distance of approximately 200 metres) by haemoglobin estimation in a finger prick blood sample $(\approx 150 \mu \mathrm{L})$ collected in a microtainer tube using a validated portable autoanalyser (Horiba ABX ES 60, Kyoto, Japan) placed in the study van. Detailed method of haemoglobin estimation is described elsewhere. ${ }^{11} \mathrm{~A}$ unique barcode will be used for linking the participant identity. 
Table 1 Information on intracluster correlation coefficient from previous studies

\section{Data source, level of clustering and} sample

\section{Intracluster correlation Average cluster size Design effect}

\begin{tabular}{|c|c|c|c|}
\hline \multicolumn{4}{|l|}{ National level data sets } \\
\hline $\begin{array}{l}\text { Comprehensive National Nutrition Survey, }{ }^{2} \\
\text { Clustering at PSU level } \\
\text { Children and adolescents: } 1-19 \text { years }\end{array}$ & $\begin{array}{l}1-4 \text { years: } 0.113 \\
\text { 5-9 years: } 0.113 \\
\text { 10-19 years: } 0.091\end{array}$ & 10 per age group & $\begin{array}{l}1-4 \text { years: } 2.28 \\
5-9 \text { years: } 2.24 \\
10-19 \text { years: } 2.20\end{array}$ \\
\hline $\begin{array}{l}\text { National Nutrition Monitoring Bureau, } \\
2012^{17} \text { (Telangana and Andhra Pradesh data), } \\
\text { Clustering at village level } \\
\text { Children under } 5 \text { years and their mothers }\end{array}$ & 0.117 & 38 & 5.3 \\
\hline
\end{tabular}

Previous published studies

\begin{tabular}{|c|c|c|c|}
\hline $\begin{array}{l}\text { Shet et } \text { al }^{18} \\
\text { C-RCT in } 12-59 \text { months children, } \\
\text { Clustering at village level }\end{array}$ & 0.05 & 25 & 2.2 \\
\hline $\begin{array}{l}\text { Bharti et al }{ }^{19} \\
\text { C-RCT in rural women and adolescent girls, } \\
\text { Clustering at village level }\end{array}$ & 0.027 & 33 & 1.92 \\
\hline \multicolumn{4}{|c|}{ Unpublished data from other studies by investigators } \\
\hline $\begin{array}{l}\text { Andhra Pradesh Children and Parents Study } \\
\text { data: household members: Household } \\
\text { members from } 29 \text { villages, age range 5-50 } \\
\text { years, } \\
\text { Clustering at village level }\end{array}$ & ICC: 0.016 & 185 & 4.036 \\
\hline
\end{tabular}

c-RCT, cluster randomised controlled trial; ICC, intracluster correlation ; PSU, Primary Sampling Unit.

Based on the haemoglobin values, participants will be classified into different grades of anaemia (or no anaemia) and will be provided IFA treatment or prophylaxis as per the Anaemia Mukt Bharat guidelines (table 2). ${ }^{42}$ The IFA dosages will be delivered in three batches (30 days at a time) for a total of 90 days. The first batch of tablets/ syrups for the household members will be handed over by the study staff soon after the screening to avoid delay. The next two batches of medication will be supplied to the participants by the respective ASHAs during their visits to the households.

Prior to start of the IFA treatment, deworming tablets (albendazole $400 \mathrm{mg}$ for adults and $200 \mathrm{mg}$ for children) will be supplied to all participants except pregnant women and school-going children who have consumed the tablet in the past 6 months through an ongoing national deworming programme (table 2 ). The participants will be instructed to consume the prescribed IFA as

Table 2 Recommended doses of iron-folic acid treatment

\begin{tabular}{|c|c|c|}
\hline Population & Prophylactic & $\begin{array}{l}\text { Treatment of mild and moderate } \\
\text { anaemia }\end{array}$ \\
\hline Children 6-59 months & $\begin{array}{l}20 \text { mg iron }+100 \text { mcg folic acid two times } \\
\text { in a week }\end{array}$ & $3 \mathrm{mg} / \mathrm{kg}$ body weight/day \\
\hline $5-9$ years & $45 \mathrm{mg}$ iron $+400 \mathrm{mcg}$ folic acid/week & $\begin{array}{l}3 \mathrm{mg} / \mathrm{kg} \text { body weight/day } \\
\text { Body weight up to } 18.5 \mathrm{~kg}: 45 \mathrm{mg} \\
\text { iron }+400 \mathrm{mcg} \text { folic acid/day } \\
\text { Body weight }>18.5 \mathrm{~kg}: 60 \mathrm{mg} \text { iron }+500 \\
\text { mcg folic acid/day }\end{array}$ \\
\hline 10-19 years (girls) and boys & $60 \mathrm{mg}$ iron $+500 \mathrm{mcg}$ folic acid/week & $120 \mathrm{mg}$ iron $+1 \mathrm{mg}$ folic acid \\
\hline Pregnant women & $60 \mathrm{mg}$ iron $+500 \mathrm{mcg}$ folic acid/day & $120 \mathrm{mg}$ iron $+1 \mathrm{mg}$ folic acid \\
\hline $\begin{array}{l}\text { Non-pregnant non-lactating women and } \\
\text { lactating women }\end{array}$ & 60 mg iron +500 mcg folic acid & 120 mg iron +1 mg folic acid \\
\hline
\end{tabular}

Severely anaemic participants will be referred to local primary health centre.

For children with severe acute malnutrition, if the child is in rehabilitation stage and has appetite and is gaining body weight, iron-folic acid dose @ $3 \mathrm{mg} / \mathrm{kg} /$ day iron will be given. 
a single dose daily after the main meals (preferably lunch) in order to reduce gastric irritation. For children below 5 years, the primary caregiver will be trained to use an autodispenser for administration of IFA syrup. The treatment regimen has been simplified by using mono-cartons for a month's supply and colour coding the medication for different dosages (online supplementary figure 1). Local language script and pictures are used for key identifiers of the blister packs (eg, the tablet, the age group for which the dose is intended). Within 1 week of supplying IFA, the participants will be contacted over the phone to seek information on side effects. Those who report discontinuation of the treatment due to side effects will be contacted by the field medical officer for further evaluation and counselling. The participants will be instructed not to consume IFA from other sources or vitamin $\mathrm{C}$ tablets or syrups simultaneously during the intervention period.

The IFA tablets and syrups with an expiry period of at least 1 year will be procured from Cyano Pharma Private Limited (Indore, India). The IFA tablets and syrups will be stored at the study centre (Indian Council of Medical Research-National Institute of Nutrition (ICMR-NIN)) in a cold room with limited access. The batchwise expiry date of the IFA medications will be recorded and will be distributed following a 'first-in-first-out' inventory method. Expired medications will be disposed as per the institutional biomedical waste management rules.

\section{Measures to enhance uptake of the intervention and its}

\section{compliance: behaviour change communication}

Lack of awareness on anaemia reduces the community uptake of the programme. ${ }^{8}$ We, therefore, plan to implement an awareness campaign based on the health belief model which has five attributes: perceived susceptibility, perceived severity, perceived benefits, perceived barriers and self-efficacy. The purpose-made tools (three videos, posters and a calendar) will be pretested among 30 women in a village to ascertain six attributes: attractiveness, comprehension, acceptance, relevance, motivation, improvement. ${ }^{13}$ Additionally, we will use interactive voice response system to enhance compliance through reminders about consumption of the tablet/syrup and messages to continue with the treatment. In a subsample of participants, we will administer a validated structured questionnaire along with baseline and endline data collection to assess the impact of behaviour change communication on health beliefs related to anaemia screening and treatment.

\section{Customised software for coordinating intervention delivery and data collection}

Customised software designed by the technical partner, Kavin Corporation, Bangalore, India, includes six components: (1) Application for data input; (2) Integration of the participant demographic data with autoanalyser reports; (3) Electronic decision support system (EDSS) to generate messages on the IFA dosages for ASHAs; (4) Near participant-navigation for follow-up of study participants; (5) Application for data collection related to process and impact evaluation (6) Dynamic dashboard for monitoring (figure 2). The EDSS is based on an algorithm designed as per the Anaemia Mukt Bharat guidelines on IFA dosages as per the grade of anaemia for different age groups (table 2). Details about the development and functions of the software will be published separately.

\section{Strengthening the skills of the ASHAs}

The ASHAs will be trained to optimise service delivery. The training modules will cover aspects such as awareness on anaemia as a public health problem, digital data collection for compliance assessment, accessing the messages through EDSS to deliver the IFA treatment and counselling. The training will be evaluated using the attributes of reaction, learning and behaviour as per the Kirkpatrick's model. $^{14}$

\section{Control group}

No active intervention will be delivered by the study team to the control group but the participants will receive benefits of the currently operational government anaemia control programme. Guidelines of the ongoing programme are provided in online supplementary table 2. However, our formative research in the study area shows that the programme is currently implemented only for the pregnant women. Data on baseline haemoglobin concentrations will not be collected in the control group because anaemia screening is a part of the intervention. Screening needs to be followed by standard of care treatment for those found to have anaemia as per the ethical guidelines. However, to evaluate balance between the two arms in terms of socio-demographic characteristics and population mean haemoglobin at baseline, $10 \%$ of the households in the control arm will undergo haemoglobin estimation followed by referral for treatment as needed. These participants will not be excluded from the final analysis. The control group participants found to be anaemic at the endline will be treated with IFA using the same protocol as the intervention group after completion of the trial.

\section{Formative research undertaken}

We conducted extensive formative research in the study area to understand the population perceptions about anaemia and its control, ground-level implementation of anaemia control programme, population dynamics including the barriers and influencers for enhancing uptake of the programme. Qualitative data collection techniques such as focus group discussions, in-depth interviews and key informant interviews were used for this data collection. We also mapped community assets including community-driven voluntary groups to understand the available facilities in the study area and their accessibility. 




Figure 2 The components of custom software to facilitate service delivery and monitoring. SMS, short messaging service.

Data collection

Household and individual level information

Household level information on demographic details, household diet diversity and assets will be collected using structured questionnaires from one or more adult household members having adequate knowledge. The respondent for the household diet diversity will usually be a woman who cooks food or a person who purchases food for the household. Individual details of eligible household members will be collected from them using a short interview questionnaire which will take 10-15 min per person for completion (table 3 , online supplementary table 3). The data collection will be done in a customised application in the tablet computer.

All enrolled participants will have their weights and heights measured by digital weighing scale (Seca 880 , $\mathrm{GmbH}$, Germany) to the nearest $100 \mathrm{~g}$ and stadiometer (Seca 213, GmbH, Germany) to the nearest $0.1 \mathrm{~cm}$, respectively. Infant length will be measured by infantometer to the nearest $0.1 \mathrm{~cm}$ (Seca 417, GmbH, Germany).

\section{Follow-up}

ASHAs will be supplied tablet computers with a preinstalled application which will be used for data collection during follow-up. During home visits, the ASHA will record compliance to IFA and any side effects/adverse effects reported by the participants in intervention group using a structured questionnaire. For compliance assessment, she will count the number of tablets/quantity of syrup consumed by inspecting the leftover tablets or syrup in the blister packs or the bottle. She will also counsel the participants to enhance medication adherence. Short videos explaining the need for IFA treatment will be circulated to the participants through their mobile phones. A follow-up evaluation of compliance will be done by the field staff at the end of 3 months. For the control group participants, information on haemoglobin testing done at a health facility and any treatment sought for anaemia will be recorded once at 3 months by ASHAs and once at 6 months by the study staff. Any participant found to have an acute illness during this follow-up visit will be referred to the nearest government health facility and their visits will be facilitated by the field staff.

\section{Participant timeline}

The trial had a preparatory phase of 10 months for formative research. The duration of intervention is 3 months. The outcome evaluation will be done at 6 months. This will also allow the participants to complete the dose in case of delayed start of treatment and also help us understand the sustainability of increment in haemoglobin.

\section{Evaluation}

\section{Outcome ascertainment}

Participants in both groups will be visited by the study team at 6 months. The endline data collection will include questionnaires to assess the compliance to IFA intervention and side effects in order to verify the ASHA-reported 
Table 3 Components of data collection at baseline

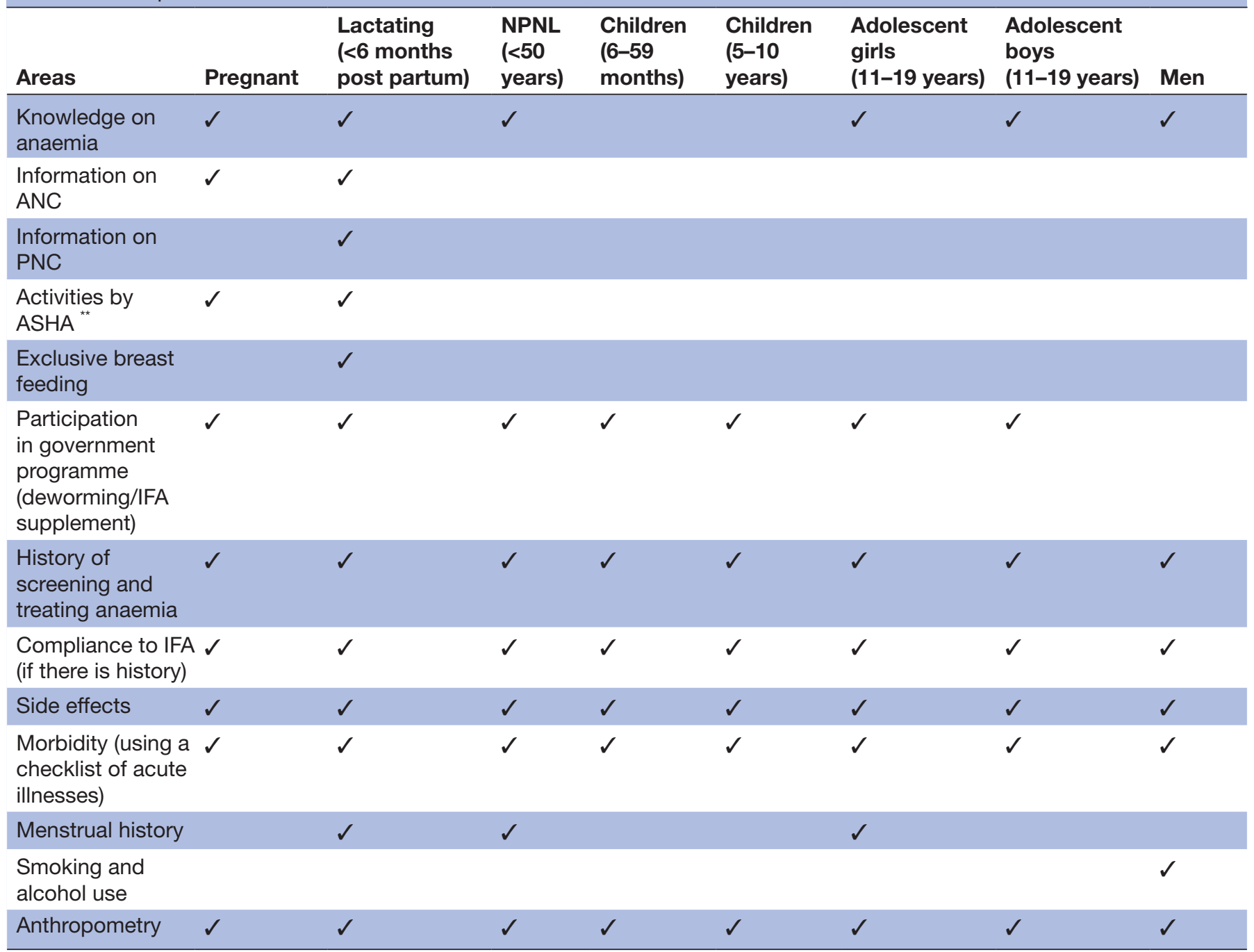

ANC, antenatal care; ASHA, Accredited Social Health Activist ; NPNL, non-pregnant non-lactating; PNC, postnatal care; IFA supplement, iron-folic acid supplementation.

compliance. Additionally, haemoglobin estimation will be carried out in all the participants from the intervention as well as control arms.

Consumption of $>50 \%$ of the total IFA recommended doses will be considered as acceptable compliance.

\section{Process evaluation}

A theory-based process evaluation will be conducted parallel to the intervention using the framework outlined by the Medical Research Council. ${ }^{15}$ Most of the process variables are embedded within the digital data collection and customised software. For collecting data on additional process variables and for impact evaluation, we will recruit separate teams to reduce bias. Once the data collection is completed for each household, the data will be uploaded to the server located at ICMR-NIN.

Additionally, we will form a 'programme learning team' focusing on the key activities of the programme. This team will conduct in-depth interviews among participants with high and low compliance to the intervention to understand the facilitators and barriers for intervention uptake. Interviews will be conducted in Telugu using a semi-structured interview guide. This procedure will be carried out in randomly selected clusters until information saturation (table 4).

Qualitative narrative data captured with digital audio recorders will be transcribed, translated, coded and analysed using NVivo, V.12.

\section{Criteria for discontinuation}

A participant will be considered to have discontinued when he/she has not taken even a single dose of the prescribed IFA medication and refuses to take tablets in future or refuses to provide any information on compliance during ASHA's visit for data collection. Common reasons for discontinuation may include out-migration from the study area, discomfort due to side effects and lack of interest.

A structured proforma will be used to report any adverse events that may occur during the study period. In case of 
Table 4 Month wise evaluation plan in intervention and control groups

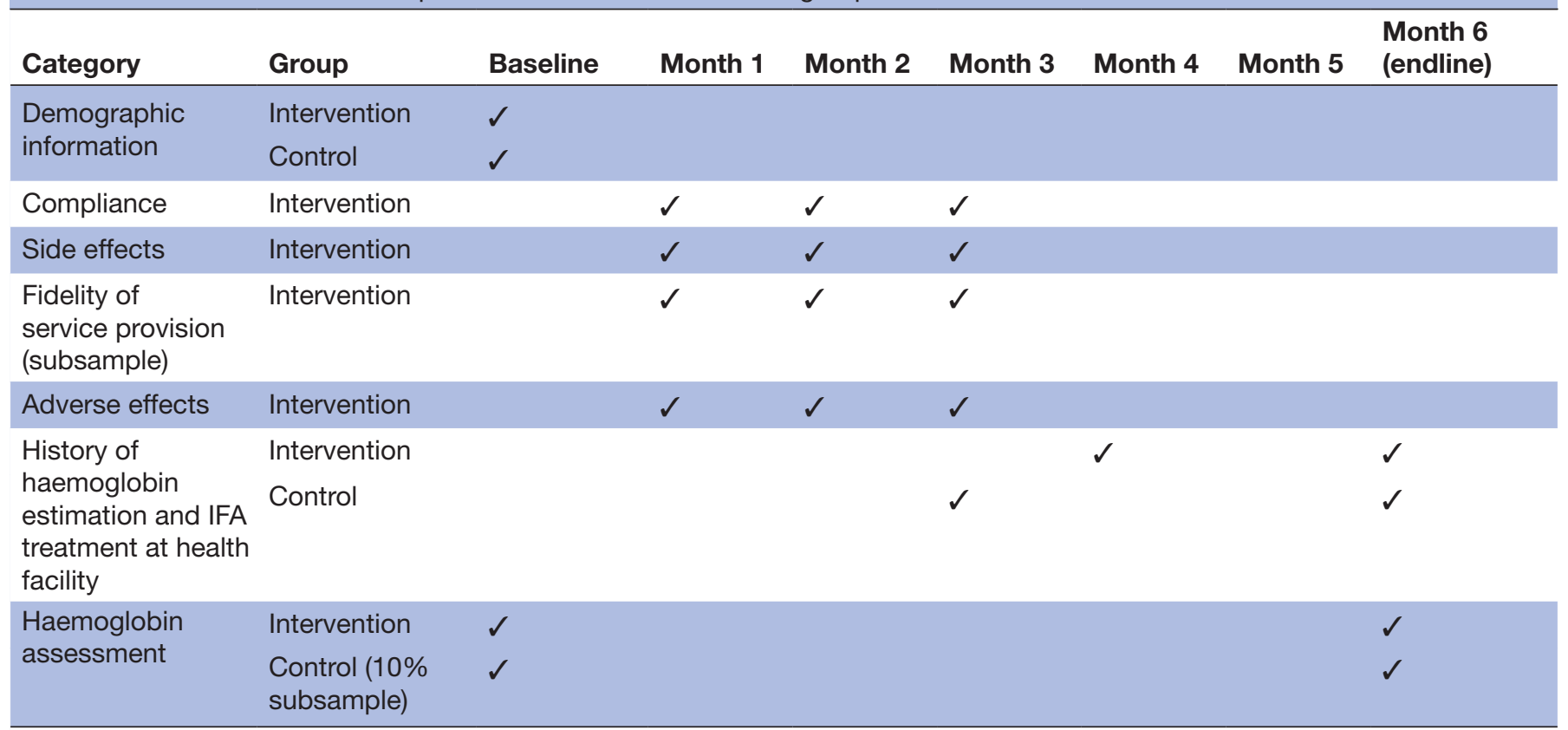

IFA, iron-folic acid.

discontinuation by a participant, the field investigator will ensure that the complete summary of the case and justification for termination is reported.

\section{Monitoring, supervision and quality control}

Regular on-site monitoring visits will be made by the supervisory staff throughout the data collection period. In addition, a real-time dashboard will be maintained for day-to-day monitoring of the data collection by the investigators.

\section{Data management and quality control}

The customised software will have inbuilt quality control mechanisms such as the 'mandatory field' option, logical entry checks with field restrictions, fixing of plausible ranges, skip logics, limited data edit and tracking of haematology quality control data. Apart from ensuring data quality, it will ensure that missing data are minimal. Data security features (Samsung Knox) will be installed in the tablet devices supplied to the field staff and ASHA workers to ensure data safety. The collected data will be encrypted for identifier variables and will be hosted in a server placed at ICMR-NIN. The software is also enabled with offline data collection.

\section{Data analysis}

Quantitative data

SPSS V.24.0 (IBM SPSS) will be used for all statistical analyses. Cluster-wise socio-demographic characteristics of the enrolled participants will be assessed to determine the balance between two arms. For clusters allocated to intervention arm, we will describe the coverage of the screening and treatment intervention, compliance with treatment and results of the initial and follow-up haemoglobin levels.

The main analyses will be 'intention-to-treat'. Perprotocol analysis will also be done. Appropriate imputation methods will be used for missing values. The primary outcome, difference in population mean haemoglobin concentration in the two arms at endpoint, will be measured using mixed/multilevel models adjusted for the cluster effect with least significant difference as post-hoc test. Risk estimates for anaemia will be assessed using forward conditional logistic regression analysis of haemoglobin. For categorical data (for secondary outcomes), generalised estimation equations will be used for comparing the proportions across the groups. The models will be presented with and without adjustment as appropriate. Analyses will also be done on the individual level data, allowing for the clustering between participants of the same age group rather than on the cluster level summarised data. As the cluster designs have two sources of variance (intra and inter cluster variability) leading to an increase in the variance, both will be taken into account in the analysis.

Effect sizes will be calculated as the raw difference between group means (absolute effect size), as well as standardised measures of effect, to transform the effect to an easily understood scale. Statistical tests and CIs will be two-sided. Between-group comparisons will be presented with $95 \%$ CIs wherever possible. The statistical significance will be set at $5 \%$.

\section{Qualitative data}

The interview data will be transcribed verbatim in Telugu followed by translation in English. About $10 \%$ of the 
translated scripts will be back-translated to Telugu for validation. Analysis will be carried out using NVivo software (V.12 plus) by two researchers trained in qualitative research, independently, using a mixed approach. Any disagreement between them will be resolved through discussion or with the help of a third reviewer.

\section{Risk mitigation plan}

A risk register has been prepared to identify the potential risks and mitigation strategies. Side effects associated with IFA supplementation (such as constipation, nausea, vomiting, diarrhoea, allergy, black stools and gastritis) are likely to be the most common risks. To minimise these, the participants will be instructed to ingest the tablets within 30 min after a meal. The information on side effects is collected in three ways: (1) A phone call from study team to participants after completion of 1 week of IFA supplementation. (2) The side effects reported by the participants through interactive voice response system (IVRS). (3) Monthly follow-up by ASHA includes data collection on side effects.

If the side effects are not mild in nature, the site medical officer will evaluate and guide future course of action. The mitigation of the risk of accidental overdose, especially by children, is addressed by: (a) using a warning label on the IFA pack; (b) detailed information on storage in a video which will be used by ASHAs to counsel the caregivers during their home visits.

\section{Funding and study oversight}

The study is funded by the ICMR and implemented by the ICMR-NIN, Hyderabad, India. ICMR-NIN is located at a distance of 25-40 kilometres from the study villages. The trial is being guided by technical advisory group and monitored by an independent Data Safety Monitoring Board (DSMB) constituted by the ICMR (online supplementary table 4 and 5). The members include public health experts, statisticians and a clinician. These committees are independent from the sponsor and competing interests.

The DSMB has reviewed the protocol and will monitor the safety of the intervention and assess the progress of the trial. Interim analyses will be conducted as per the statistical analytical plan endorsed by the DSMB. The board will review the data two times a year. Any adverse events reported in the intervention arm will be documented and all severe adverse events and protocol amendments will be reported to the ethics committee and the DSMB.

\section{Ethics and dissemination}

The study protocol conforms to the Standard Protocol Items: Recommendations for Interventional Trials guidelines. The trial is approved by the Institutional Ethics Committee (IRB. No. 08/I/2018) and is registered with the Clinical Trials Registry of India. Written informed consent (thumb print in case of illiterate persons) will be obtained from participants by the project staff before enrolment. For participants below 19 years, informed consent will be obtained from parents. For children aged 7-19 years, assent will also be obtained. The study results will be disseminated to the local and national government health departments for probable programme modifications. The study results will also be disseminated through academic channels like national conferences, publishing as full-length papers in peer-reviewed scientific journals.

\section{Data sharing}

The data will be shared as per the ICMR guidelines (currently under development) at the time of completion of the study. Restricted data sets will be shared by the corresponding author in conformity with the regulatory guidelines on reasonable request.

\section{Significance and impact}

The interventions planned as a part of the STAR trial, being well aligned with the nationwide Anaemia Mukt Bharat programme in India, have potential for immediate scale up. ${ }^{4}$ Additionally, the intervention includes several novel components such as POC testing of anaemia by a precise automated haematology analyser, customised software for coordinating the study activities to enhance efficiency, simplified intervention delivery for the ASHAs and strong behaviour change communication campaign. If found effective, the STAR strategy will pave the way for future programmes for targeted and efficient anaemia control.

A noteworthy feature of the study is inclusion of men below 50 years of age, a group which is usually not targeted by anaemia control programmes. However, one in four men are likely to be anaemic as reported by the fourth round of NFHS ${ }^{16}$ and treating them will not only enhance their economic productivity but would also increase overall acceptability of the intervention due to their influence on the household decisions. Other important aspects of the study include rigorous training of ASHAs and optimisation of intervention delivery by them through simplified IFA packaging and customised videos. Our formative research among the ASHAs revealed that although motivated to provide intervention delivery, ASHAs have limited knowledge on treatment of anaemia and lack tools for counselling the beneficiaries to augment the uptake and compliance of IFA supplements. The STAR trial intervention is optimised to address this important issue. Moreover, the customised software has several features to facilitate effective intervention delivery by the ASHAs and the study plans to implement measures to enhance compliance such as use of IVRS as dose reminders to the participants. With wide penetration of mobile phones in rural areas, utility of such high frequency messaging may be high; the present study offers an opportunity of its evaluation.

Our study thus has futuristic components to make the anaemia control programme more effective apart from its potential to generate evidence on the impact of 'screen and treat' approach. While developing the intervention, we have been mindful of potential scalability. The 
household level screening can be taken up by the health departments that are already implementing screening programmes for other health problems. The software for integration of 'screen and treat' activities will be shared with the government agencies based on request. If the intervention is found to be effective, discussions will be initiated with the relevant health authorities to encourage adoption and scale up of the study intervention.

Acknowledgements The authors wish to acknowledge Late Dr Maharaj K Bhan, former Chairperson, Technical Advisory Group (TAG), for his scientific mentorship and are thankful to the TAG members: Dr Prema Ramachandran, Nutrition Foundation of India, New Delhi; Dr Ravindra Mohan Pandey, All India Institute of Medical Sciences, New Delhi; Dr Sila Deb, Ministry of Health and Family Welfare, New Delhi and Dr L Jeyaseelan, Christian Medical College, Vellore, for their valuable input on the study design.

Collaborators None declared.

Contributors BK and RPu: conceptualised and developed the study design with input from ASP and HSS. LFA: developed study protocols and wrote the first draft along with BK. TD and RPa: developed the POC screening method and protocol. ASP and SKB contributed to development of study design and methodology. HSS provided critical intellectual input for improving the study design and manuscript. All the authors reviewed and approved the final version of the manuscript and agree to be accountable for all aspects of the work.

Funding This work was supported by Indian Council of Medical Research (grant number 5/7/1581/2018-RBMH\&CH), V. Ramalingaswami Bhawan, Ansari Nagar, New Delhi, India.

Competing interests None declared.

Patient consent for publication Not applicable.

Provenance and peer review Not commissioned; externally peer reviewed.

Data availability statement The data will be shared as per the ICMR Guidelines (currently under development) at the time of completion of the study. Restricted data sets will be shared by the corresponding author in conformity with the regulatory guidelines upon reasonable request.

Supplemental material This content has been supplied by the author(s). It has not been vetted by BMJ Publishing Group Limited (BMJ) and may not have been peer-reviewed. Any opinions or recommendations discussed are solely those of the author(s) and are not endorsed by BMJ. BMJ disclaims all liability and responsibility arising from any reliance placed on the content. Where the content includes any translated material, BMJ does not warrant the accuracy and reliability of the translations (including but not limited to local regulations, clinical guidelines, terminology, drug names and drug dosages), and is not responsible for any error and/or omissions arising from translation and adaptation or otherwise.

Open access This is an open access article distributed in accordance with the Creative Commons Attribution Non Commercial (CC BY-NC 4.0) license, which permits others to distribute, remix, adapt, build upon this work non-commercially, and license their derivative works on different terms, provided the original work is properly cited, appropriate credit is given, any changes made indicated, and the use is non-commercial. See: http://creativecommons.org/licenses/by-nc/4.0/.

ORCID iDs

Bharati Kulkarni http://orcid.org/0000-0003-0636-318X

Anju Sinha Pradhan http://orcid.org/0000-0002-0830-7723
REFERENCES

1 Haas JD, Brownlie T, Tt B. Iron deficiency and reduced work capacity: a critical review of the research to determine a causal relationship. J Nutr 2001;131:676S-90. discussion 88S-90S.

2 Ministry of Health and Family Welfare Government of India. Comprehensive national nutrition survey (CNNS) national report. New Delhi: Ministry of Health and Family Welfare Government of India, UNICEF, Population Council, 2018.

3 International Institute for Population Sciences. National family health survey (NFHS-4) 2015-16 India. IIPS-Mumbai: International Institute for Population Sciences, 2017: 1-192.

4 Government of India. Intensified national intensified iron plus initiative (I-NIPI) operational guidelines for programme managers. New Delhi: Ministry of Health and Family Welfare Government of India, 2018.

5 Chaudhary R, Dubey A, Sonker A. Techniques used for the screening of hemoglobin levels in blood donors: current insights and future directions 2017;8:75-88.

6 McLennan JD, Steele M. Anemia screening and treatment outcomes of children in a low-resource community in the Dominican Republic. $J$ Trop Pediatr 2016;62:116-22.

7 McCormack T, Ayub R, Aziz F, et al. Point-of-care testing facilitates screening and treatment for anaemia in women and children in rural Pakistan. Aust J Rural Health 2018;26:194-8.

8 Dasi T, Palika R, Pullakhandham R. Hemoglobin measurement in capillary blood by a portable autoanalyzer for population level screening of anemia: validation in cross-sectional and longitudinal studies. British Journal of Nutrition 2021.

9 International Institute for Population Sciences. National family health survey (2019-2020) district fact sheet Medchal Malkajgiri. Telangana: International Institute for Population Sciences, 2020.

10 International Institute for Population Sciences. District level household \& facility survey (DLHS-4). Available: http://rchiips.org/ DLHS-4.html

11 Augustine LF, Dasi T, Palika R, et al. Point of care diagnosis of anemia using portable auto analyzer. Indian Pediatr 2020;57:568-9.

12 World Health Organization. Haemoglobin concentrations for the diagnosis of anaemia and assessment of severity (No. WHO/NMH/ NHD/MNM/11.1). World Health Organization, 2011.

13 Orji R, Vassileva J, Mandryk R. Towards an effective health interventions design: an extension of the health belief model. Online J Public Health Inform 2012;4.

14 Desilets LD. An update on Kirkpatrick's model of evaluation: Part two 2018;49:292-3

15 Moore GF, Audrey S, Barker M, et al. Process evaluation of complex interventions: medical Research Council guidance. BMJ 2015;350:h1258.

16 Didzun O, De Neve J-W, Awasthi A, et al. Anaemia among men in India: a nationally representative cross-sectional study. Lancet Glob Health 2019;7:e1685-94.

17 National Nutrition Monitoring Bureau. Diet and nutritional status of rural population, prevalence of hypertension and diabetes among adults and infant and young child feeding practices. Report of third repeat survey. Technical report No: 26 . Hyderabad: National Institute of Nutrition, Indian Council of Medical Research, 2012. http://www. nnmbindia.org/1_NNMB_Third_Repeat_Rural_Survey__Technicl_ Report_26.pdf

18 Shet AS, Zwarenstein M, Mascarenhas M, et al. The Karnataka Anemia Project 2--design and evaluation of a community-based parental intervention to improve childhood anemia cure rates: study protocol for a cluster randomized controlled trial. Trials 2015;16:599.

19 Bharti S, Bharti B, Naseem S, et al. A community-based cluster randomized controlled trial of "directly observed home-based daily iron therapy" in lowering prevalence of anemia in rural women and adolescent girls. Asia Pac J Public Health 2015;27:NP1333-44. 